doi: $10.13108 / 2020-12-1-114$

\title{
UNIQUENESS THEOREMS FOR MEROMORPHIC FUNCTIONS ON ANNULI
}

\section{A. RATHOD}

\begin{abstract}
In this paper, we discuss the uniqueness problems of meromorphic functions on annuli. We prove a general theorem on the uniqueness of meromorphic functions on annuli. An analogue of a famous Nevanlinna's five-value theorem is proposed. The main result in this paper is an analog of a result on the plane $\mathbb{C}$ obtained by H.S. Gopalkrishna and Subhas S. Bhoosnurmath for an annuli. That is, let $f_{1}(z)$ and $f_{2}(z)$ be two transcendental meromorphic functions on the annulus $\mathbb{A}=\left\{z: \frac{1}{R_{0}}<|z|<R_{0}\right\}$, where $1<R_{0} \leqslant+\infty$. Let $\left.a_{j}, j=1,2, \ldots, q\right)$, be $q$ distinct complex numbers in $\overline{\mathbb{C}}$, and $k_{j}, j=1,2, \ldots, q$ be positive integers or $\infty$ satisfying

$$
k_{1} \geqslant k_{2} \geqslant \ldots \geqslant k_{q}
$$
\end{abstract}

If

$$
\bar{E}_{\left.k_{j}\right)}\left(a_{j}, f_{1}\right)=\bar{E}_{\left.k_{j}\right)}\left(a_{j}, f_{2}\right), \quad j=1,2, \ldots, q,
$$

and

$$
\sum_{j=2}^{q} \frac{k_{j}}{k_{j}+1}-\frac{k_{1}}{k_{1}+1}>2,
$$

then $f_{1}(z) \equiv f_{2}(z)$.

Keywords : Nevanlinna theory, meromorphic functions, annuli.

Subject Classification: 30D35

\section{INTRODUCTION}

The uniqueness theory of meromorphic functions is an interesting problem in the value distribution theory and as well as the uniqueness theory of algebroid functions. The uniqueness problem of algebroid functions was first considered by Valiron [3], afterwards several uniqueness theorems of algebroid functions in the complex plane $\mathbb{C}$ were proved. In 2005, A. Ya. Khrystiyanyn and A. A. Kondratyuk have proposed on the Nevanlinna Theory for meromorphic functions on annuli (see [6], [7]) and after this work, many others work in this area appeared, see [5], [12], [13], [14], [15], [21], [28]). In 2009, Cao and Yi [8] studied the uniqueness of meromorphic functions sharing some values on annuli. In 2015, Yang Tan [2], Yang Tan and Yue Wang [1] proved some interesting results on the multiple values and uniqueness of algebroid functions on annuli and also others have proved several results for algebroid functions on annuli, see [9], [11], [16], [18], [19], [20], [22], [23], [24], [25], [26], [27], [29], [30]. Thus, it is interesting to consider the uniqueness problem of algebroid functions in multiply connected domains. By Doubly connected mapping theorem [10], each doubly connected domain is conformally equivalent to the annulus $\{z: r<|z|<R\}, 0 \leqslant r<R \leqslant+\infty$. We consider only two cases : $r=0, R=+\infty$

A. RAthod, Uniqueness theorems For MERomorphic FUnCtions on ANnUli.

(C) A. RATHOD 2020 .

Поступила 4 июня 2019 2. 
simultaneously and $0 \leqslant r<R \leqslant+\infty$. In the latter case the homothety $z \mapsto \frac{z}{r R}$ reduces the given domain to the annulus

$$
\mathbb{A}=\mathbb{A}\left(R_{0}\right)=\mathbb{A}\left(\frac{1}{R_{0}}, R_{0}\right)=\left\{z: \frac{1}{R_{0}}<|z|<R_{0}\right\},
$$

where $R_{0}=\sqrt{\frac{R}{r}}$. Thus, in both cases, each annulus is invariant with respect to the inversion $z \mapsto \frac{1}{z}$.

\section{BASIC NOTATIONS IN THE NEVANLINNA THEORY ON ANNUli}

Let $f$ be a meromorphic function on the annulus $\mathbb{A}=\left\{z: \frac{1}{R_{0}}<|z|<R_{0}\right\}$. We recall classical notations of Nevanlinna theory as follows

$$
\begin{aligned}
& N(R, f)=\int_{0}^{R} \frac{n(t, f)-n(0, f)}{t} d t+n(0, f) \log R, \\
& m(R, f)=\frac{1}{2 \pi} \int_{0}^{2 \pi} \log ^{+}\left|f\left(R e^{i \theta}\right)\right| d \theta, \\
& T(R, f)=N(R, f)+m(R, f),
\end{aligned}
$$

where $\log ^{+} x=\max \{\log x, 0\}$, and $n(t, f)$ is the counting function of poles of the function $f$ in $\{z:|z| \leqslant t\}$. Let

$$
\begin{aligned}
& N_{1}(R, f)=\int_{\frac{1}{R}}^{1} \frac{n_{1}(t, f)}{t} d t, \\
& N_{2}(R, f)=\int_{1}^{R} \frac{n_{2}(t, f)}{t} d t, \\
& m_{0}(R, f)=m(R, f)+m\left(\frac{1}{R}, f\right)-2 m(1, f), \\
& N_{0}(R, f)=N_{1}(R, f)+N_{2}(R, f),
\end{aligned}
$$

where $n_{1}(t, f)$ and $n_{2}(t, f)$ are the counting functions of the poles of the function $f$ in $\{z: t<$ $|z| \leqslant 1\}$ and $\{z: 1<|z| \leqslant t\}$, respectively. The Nevanlinna charecteristic of $f$ on the annulus $\mathbb{A}$ is defined by

$$
T_{0}(R, f)=m_{0}(r, f)+N_{0}(R, f) .
$$

Definition 1 (8). Let $f(z)$ be a non-constant meromorphic function on the annulus $\mathbb{A}\left(R_{0}\right)=$ $\left\{z: 1 / R_{0}<|z|<R_{0}\right\}$, where $1<R_{0}<+\infty$. The function $f$ is called a transcendental or admissible meromorphic function on the annulus $\mathbb{A}\left(R_{0}\right)$ provided that

$$
\lim _{R \rightarrow \infty} \sup \frac{T_{0}(R, f)}{\log R}=\infty, \quad 1<R<R_{0}=+\infty
$$

or

$$
\lim _{R \rightarrow R_{0}} \sup \frac{T_{0}(R, f)}{-\log \left(R_{0}-R\right)}=\infty, \quad 1<R<R_{0}<+\infty
$$

respectively.

For a transcendental or admissible meromorphic function on the annulus $\mathbb{A}$, the identity

$$
S(R, f)=o\left(T_{0}(R, f)\right)
$$

holds for all $1<R<R_{0}$ except for the set $\triangle_{R}$ or the set $\triangle_{R}^{\prime}$ mentioned in Theorem 1 , respectively. 
Next, we have

$$
\begin{aligned}
\overline{N_{0}}\left(R, \frac{1}{f-a}\right) & =\overline{N_{1}}\left(R, \frac{1}{f-a}\right)+\overline{N_{2}}\left(R, \frac{1}{f-a}\right) \\
& =\int_{\frac{1}{R}}^{1} \frac{\overline{n_{1}}\left(t, \frac{1}{f-a}\right)}{t} d t+\int_{1}^{R} \frac{\overline{n_{2}}\left(t, \frac{1}{f-a}\right)}{t} d t
\end{aligned}
$$

in which each zero of the function $f-a$ is counted only once.

We use $\left.\bar{n}_{1}^{k}\right)\left(t, \frac{1}{f-a}\right)$, respectively, $\bar{n}_{1}\left(k\left(t, \frac{1}{f-a}\right)\right.$ to denote the counting function of poles of the function $\frac{1}{f-a}$ with the multiplicities not exceeding $k$, respectively, greater than $k$ in $\{z: t<|z| \leqslant 1\}$, where each point is counted only once. In a same way we introduce the notations

$$
{\overline{N_{1}}}^{k)}(t, f), \quad{\overline{N_{1}}}^{(k}(t, f), \quad{\overline{N_{2}}}^{k)}(t, f), \quad{\overline{N_{2}}}^{(k}(t, f), \quad{\overline{N_{0}}}^{k)}(t, f), \quad{\overline{N_{0}}}^{(k}(t, f) .
$$

The following theorem was proved in [8].

Theorem 1. (The Second Fundamental Theorem on annuli). Let $f$ be a non constant meromorphic function on the annulus $\mathbb{A}=\left\{z: \frac{1}{R_{0}}<|z|<R_{0}\right\}$, where $1<R<R_{0} \leqslant+\infty$. Let $a_{1}, a_{2}, \ldots, a_{q}$ be $q$ distinct complex numbers in the extended complex plane $\overline{\mathbb{C}}=\mathbb{C} \cup\{\infty\}$, let $k_{1}, k_{2}, \ldots, k_{q}$ be $q$ positive integers, and let $\lambda \geqslant 0$. Then

(i) $(q-2) T_{0}(R, f)<\sum_{j=1}^{q} N_{0}\left(R, \frac{1}{f-a_{j}}\right)-N_{0}^{(1)}(R, f)+S(R, f)$,

(ii) $(q-2) T_{0}(R, f)<\sum_{j=1}^{q} \overline{N_{0}}\left(R, \frac{1}{f-a_{j}}\right)+S(R, f)$,

(iii) $\left.(q-2) T_{0}(R, f)<\sum_{j=1}^{q} \frac{k_{j}}{k_{j}+1} \bar{N}_{0}^{k_{j}}\right)\left(R, \frac{1}{f-a_{j}}\right)+\sum_{j=1}^{q} \frac{1}{k_{j}+1} N_{0}\left(R, \frac{1}{f-a_{j}}\right)+S(R, f)$,

(iv) $\left.\left(q-2-\sum_{j=1}^{q} \frac{1}{k_{j}+1}\right) T_{0}(R, f)<\sum_{j=0}^{q} \frac{k_{j}}{k_{j}+1}{\overline{N_{0}}}^{k_{j}}\right)\left(R, \frac{1}{f-a_{j}}\right)+S(R, f)$,

where

$$
N_{0}^{(1)}(R, f)=N_{0}\left(R, \frac{1}{f^{\prime}}\right)+2 N_{0}(R, f)-N_{0}\left(R, f^{\prime}\right)
$$

and it holds: 1 . In the case $R_{0}=+\infty$

$$
m_{0}\left(R, \frac{f^{\prime}}{f}\right)=O\left(\log \left(R T_{0}(R, f)\right)\right)
$$

for $R \in(1,+\infty)$ except for the set $\triangle_{R}$ such that

$$
\int_{\triangle_{R}} R^{\alpha-1} d R<+\infty
$$

2. In the case $R_{0}<+\infty$

$$
m_{0}\left(R, \frac{f^{\prime}}{f}\right)=O\left(\log \left(\frac{T_{0}(R, f)}{R_{0}-R}\right)\right)
$$


for $R \in\left(1, R_{0}\right)$ except for the set $\triangle_{R}^{\prime}$ such that

$$
\int_{\triangle_{R}^{\prime}} \frac{d R}{\left(R_{0}-R^{\alpha-1}\right)}<+\infty
$$

\section{MAIN ResUlts}

Let $f(z)$ be a meromorphic function on the annulus $\mathbb{A}=\left\{z: \frac{1}{R_{0}}<|z|<R_{0}\right\}$, where $1<R<R_{0} \leqslant+\infty$, and $a$ be a complex number in the extended complex plane $\overline{\mathbb{C}}=\mathbb{C} \cup\{\infty\}$. We denote $E(a, f)=\{z \in \mathbb{A}: f(z)-a=0\}$, where each zero with multiplicity $m$ is counted $m$ times. If we ignore the multiplicity, then the set is denoted by $\bar{E}(a, f)$. We use $\bar{E}_{k)}(a, f)$ to denote the set of zeros of $f-a$ with multiplicities no greater than $k$, in which each zero is counted only once.

Our main result below is an analog of a result on the plane $\mathbb{C}$ obtained by H. S. Gopalkrishna and Subhas S. Bhoosnurmath [4].

Theorem 2. Let $f_{1}(z)$ and $f_{2}(z)$ be two transcendental meromorphic functions on the annulus $\mathbb{A}=\left\{z: \frac{1}{R_{0}}<|z|<R_{0}\right\}$, where $1<R_{0} \leqslant+\infty$. Let $a_{j}(j=1,2, \ldots, q)$ be $q$ distinct complex numbers in $\overline{\mathbb{C}}$, and $k_{j}, j=1,2, \ldots, q$ be positive integers or $\infty$ satisfying

$$
k_{1} \geqslant k_{2} \geqslant \ldots \geqslant k_{q}
$$

If

$$
\bar{E}_{\left.k_{j}\right)}\left(a_{j}, f_{1}\right)=\bar{E}_{\left.k_{j}\right)}\left(a_{j}, f_{2}\right), \quad j=1,2, \ldots, q,
$$

and

$$
\sum_{j=2}^{q} \frac{k_{j}}{k_{j}+1}-\frac{k_{1}}{k_{1}+1}>2
$$

then $f_{1}(z) \equiv f_{2}(z)$.

Proof. We assume that $a_{j}, j=1,2, \ldots, q$, are finite complex numbers, otherwise we make a suitable Mobius transformation. By Theorem 1 we have

$$
\begin{aligned}
(q-2) T_{0}\left(R, f_{1}\right)< & \left.\sum_{j=1}^{q} \frac{k_{j}}{k_{j}+1} \overline{N_{0}}{ }^{k_{j}}\right)\left(R, \frac{1}{f_{1}-a_{j}}\right) \\
& +\sum_{j=1}^{q} \frac{1}{k_{j}+1} T_{0}\left(R, f_{1}\right)+S\left(R, f_{1}\right) .
\end{aligned}
$$

This implies

$$
\left.\left(q-2-\sum_{j=1}^{q} \frac{1}{k_{j}+1}\right) T_{0}\left(R, f_{1}\right)<\sum_{j=1}^{q} \frac{k_{j}}{k_{j}+1}{\overline{N_{0}}}^{k_{j}}\right)\left(R, \frac{1}{f_{1}-a_{j}}\right)+S\left(R, f_{1}\right) .
$$

Therefore,

$$
\left(\sum_{j=1}^{q} \frac{k_{j}}{k_{j}+1}-2\right) T_{0}\left(R, f_{1}\right)<\sum_{j=1}^{q} \frac{k_{j}}{k_{j}+1} \bar{N}_{0}^{\left.k_{j}\right)}\left(R, \frac{1}{f_{1}-a_{j}}\right)+S\left(R, f_{1}\right) .
$$

Condition (1) implies:

$$
1 \geqslant \frac{k_{1}}{k_{1}+1} \geqslant \frac{k_{2}}{k_{2}+1} \geqslant \ldots \geqslant \frac{k_{q}}{k_{q}+1} \geqslant \frac{1}{2}
$$


It follows from the above inequalities and (4) that

$$
\left.\left(\sum_{j=1}^{q} \frac{k_{j}}{k_{j}+1}-2\right) T_{0}\left(R, f_{1}\right)<\frac{k_{1}}{k_{1}+1} \sum_{j=1}^{q} \overline{N_{0}} k_{j}\right)\left(R, \frac{1}{f_{1}-a_{j}}\right)+S\left(R, f_{1}\right) .
$$

In the same way,

$$
\left.\left(\sum_{j=1}^{q} \frac{k_{j}}{k_{j}+1}-2\right) T_{0}\left(R, f_{2}\right)<\frac{k_{1}}{k_{1}+1} \sum_{j=1}^{q} \overline{N_{0}} k_{j}\right)\left(R, \frac{1}{f_{2}-a_{j}}\right)+S\left(R, f_{2}\right) .
$$

Since $f_{1}(z) \not \equiv f_{2}(z)$, it follows from $(2)$ that

$$
\begin{aligned}
\left.\left.\max \left(\sum_{j=1}^{q}{\overline{N_{0}}}^{k_{j}}\right)\left(R, \frac{1}{f_{1}-a_{j}}\right), \sum_{j=1}^{q}{\overline{N_{0}}}^{k_{j}}\right)\left(R, \frac{1}{f_{2}-a_{j}}\right)\right) & \leqslant N_{0}\left(R, \frac{1}{f_{1}-f_{2}}\right) \\
& \leqslant T_{0}\left(R, \frac{1}{f_{1}-f_{2}}\right)+O(1) \\
& \leqslant T_{0}\left(R, f_{1}\right)+T_{0}\left(R, f_{2}\right) .
\end{aligned}
$$

Therefore, from the above discussion we obtain

$$
\left(\sum_{j=1}^{q} \frac{k_{j}}{k_{j}+1}-2\right) T_{0}\left(R, f_{1}\right)<\frac{k_{1}}{k_{1}+1}\left[T_{0}\left(R, f_{1}\right)+T_{0}\left(R, f_{2}\right)\right]+S\left(R, f_{1}\right) .
$$

Similarly,

$$
\left(\sum_{j=1}^{q} \frac{k_{j}}{k_{j}+1}-2\right) T_{0}\left(R, f_{2}\right)<\frac{k_{1}}{k_{1}+1}\left[T_{0}\left(R, f_{1}\right)+T_{0}\left(R, f_{2}\right)\right]+S\left(R, f_{2}\right) .
$$

Summing two above equations, we obtain:

$$
\left(\sum_{j=2}^{q} \frac{k_{j}}{k_{j}+1}-\frac{k_{1}}{k_{1}+1}-2\right)\left[T_{0}\left(R, f_{1}\right)+T_{0}\left(R, f_{2}\right)\right]<S\left(R, f_{1}\right)+S\left(R, f_{2}\right) .
$$

By (3) we get:

$$
T_{0}\left(R, f_{1}\right)+T_{0}\left(R, f_{2}\right)<S\left(R, f_{1}\right)+S\left(R, f_{2}\right),
$$

which is impossible since $f_{1}(z)$ and $f_{2}(z)$ are transcendental meromorphic functions. Hence, $f_{1}(z) \equiv f_{2}(z)$. This completes the proof.

From Theorem 2, we get the following corollary.

Corollary 1. Let $f_{1}(z)$ and $f_{2}(z)$ be two transcendental meromorphic functions on the annulus $\mathbb{A}=\left\{z: \frac{1}{R_{0}}<|z|<R_{0}\right\}$, where $1<R_{0} \leqslant+\infty$. Let $a_{j}, j=1,2, \ldots, q$, be $q(\geqslant 5)$ distinct complex numbers in $\overline{\mathbb{C}}$, and $k_{j}, j=1,2, \ldots, q$, be positive integers or $\infty$ satisfying

$$
k_{1} \geqslant k_{2} \geqslant \geqslant k_{q}
$$

and

$$
\bar{E}_{\left.k_{j}\right)}\left(a_{j}, f_{1}\right)=\bar{E}_{\left.k_{j}\right)}\left(a_{j}, f_{2}\right), \quad j=1,2, \ldots, q
$$

\section{Then}

(i) if $q \geqslant 7$, then $f_{1}(z) \equiv f_{2}(z)$.

(ii) if $q=6$ and $k_{3} \geqslant 2$, then $f_{1}(z) \equiv f_{2}(z)$.

(iii) if $q=5$, and $k_{3} \geqslant 3$ and $k_{5} \geqslant 2$, then $f_{1}(z) \equiv f_{2}(z)$.

(iv) if $q=5$ and $k_{4} \geqslant 4$, then $f_{1}(z) \equiv f_{2}(z)$.

From Corollary 1, we obtain a following theorem. 
Theorem 3. Let $f_{1}(z)$ and $f_{2}(z)$ be two transcendental meromorphic functions on the annulus $\mathbb{A}=\left\{z: \frac{1}{R_{0}}<|z|<R_{0}\right\}$, where $1<R_{0} \leqslant+\infty$. Let $a_{j}, j=1, \ldots, 7$, be five distinct complex numbers in $\overline{\mathbb{C}}$. If $\bar{E}\left(a_{j}, f_{1}\right)=\bar{E}\left(a_{j}, f_{2}\right)$ for $j=1, \ldots, 7$, then $f_{1}(z) \equiv f_{2}(z)$.

Corollary 1 implies the following analogue of Nevanlinna's five value theorem. In the case $R_{0}=+\infty$, this statement was proved by Kondratyuk and Laine [31].

Theorem 4. Let $f_{1}(z)$ and $f_{2}(z)$ be two transcendental meromorphic functions on the annulus $\mathbb{A}=\left\{z: \frac{1}{R_{0}}<|z|<R_{0}\right\}$, where $1<R_{0} \leqslant+\infty$. Let $a_{j}, j=1, \ldots, 5$, be five distinct complex numbers in $\overline{\mathbb{C}}$. If $\bar{E}\left(a_{j}, f_{1}\right)=\bar{E}\left(a_{j}, f_{2}\right)$ for $j=1, \ldots, 5$, then $f_{1}(z) \equiv f_{2}(z)$.

The condition of $f_{1}(z)$ and $f_{2}(z)$ share five values in Theorem 3.3 can not be weakened to that $f_{1}(z)$ and $f_{2}(z)$ share four values. For example, the functions $f_{1}(z)=e^{z}$ and $f_{2}(z)=e^{-z}$ share four values $0,1,-1, \infty$, but $f_{1}(z) \neq f_{2}(z)$.

\section{BIBLIOGRAPHY}

1. Yang Tan, Yue Wang. On the multiple values and uniqueness of algebroid functions on annuli // Compl. Variab. Ell. Equat. 60:9, 1254-1269 (2015).

2. Yang Tan. Several uniqueness theorems of algebroid functions on annuli // Acta Mathematica Scientia. 36:1, 295-316 (2016).

3. Valiron G. Sur quelques proprietes des fonctions algebroldes // C.R. Math. Acad. des Sci. Paris, 189, 824-826 (1929).

4. H.S. Gopalkrishna, S.S. Bhoosnurmath. Uniqueness theorems for meromorphic functions // Math. Scand. 39, 125-130 (1976).

5. M.L. Fang. A note on a result of Singh and Kulkarni // Int. J. Math. Sci. 23:4, 285-288 (2000).

6. A.Ya. Khrystiyanyn, A.A. Kondratyuk. On the Nevanlinna theory for meromorphic functions on annuli. I // Mathematychni Studii. 23:1, 19-30 (2005).

7. A.Ya. Khrystiyanyn, A.A. Kondratyuk. On the Nevanlinna theory for meromorphic functions on annuli. II // Mathematychni Studii, 24:2, 57-68 (2005).

8. T.B. Cao, H.X. Yi, H.Y. Xu. On the multiple values and uniqueness of meromorphic functions on annuli // Comput. Math. Appl. 58:7, 1457-1465 (2009).

9. Yu-Zan He and Xiu-Zhi Xiao. Algebroid functions and ordinarry difference equations. Beijing, Science Press (1988).

10. A. Fernandez. On the value distribution of meromorphic function in the punctured plane // Mathematychni Studii. 34:2, 136-144 (2010).

11. Meili Liang. On the value distribution of algebroid functions // SOP Trans. Appl. Math. 1:1, 23-30 (2014).

12. W.K. Hayman. Meromorphic functions. Oxford University Press, Oxford (1964).

13. R.S. Dyavanal, A. Rathod. Uniqueness theorems for meromorphic functions on annuli // Indian J. Math. Math. Sci. 12:1, 1-10 (2016).

14. R.S. Dyavanal, A. Rathod. Multiple values and uniqueness of meromorphic functions on annuli // Int. J. Pure Appl. Math. 107:4, 983-995 (2016).

15. R.S. Dyavanal, A. Rathod. On the value distribution of meromorphic functions on annuli // Indian J. Math. Math. Sci. 12:2, 203-217 (2016).

16. R.S. Dyavanal, A. Rathod. Some generalisation of Nevanlinna's five-value theorem for algebroid functions on annuli// Asian J. Math. Comp. Research. 20:2, 85-95 (2017).

17. R.S. Dyavanal, A. Rathod. Nevanlinna's five-value theorem for derivatives of meromorphic functions sharing values on annuli // Asian J. Math. Comp. Research. 20:1, 13-21 (2017).

18. R.S. Dyavanal, A. Rathod. Unicity theorem for algebroid functions related to multiple values and derivatives on annuli // Int. J. Fuzzy Math. Arch. 13:1, 25-39 (2017).

19. R.S. Dyavanal, A. Rathod. General Milloux inequality for algebroid functions on annuli// Int. J. Math. Appl. 5:3, 319-326 (2017). 
20. A. Rathod. The multiple values of algebroid functions and uniqueness// Asian Journal of Mathematics and Computer Research, 14:2, 150-157(2016).

21. Ashok Rathod. The uniqueness of meromorphic functions concerning five or more values and small functions on annuli // Asian J. Current Research. 1:3, 101-107 (2016).

22. A. Rathod. Uniqueness of algebroid functions dealing with multiple values on annuli// J. Basic Appl. Research Int. 19:3, 157-167 (2016).

23. A. Rathod. On the deficiencies of algebroid functions and their differential polynomials// Journal of Basic and Applied Research International, 1:1, 1-11(2016).

24. A. Rathod. The multiple values of algebroid functions and uniqueness on annuli // Konuralp J. Math. 5:2, 216-227 (2017).

25. A. Rathod. Several uniqueness theorems for algebroid functions // J. Anal. 25:2, 203-213 (2017).

26. A. Rathod. Nevanlinna's five-value theorem for algebroid functions // Ufa Math. J. 10:2, 127-132 (2018).

27. A. Rathod. Nevanlinna's five-value theorem for derivatives of algebroid functions on annuli // Tamkang J. Math. 49:2, 129-142 (2018).

28. S.S. Bhoosnurmath, R.S. Dyavanal, Mahesh Barki, A. Rathod. Value distribution for n'th difference operator of meromorphic functions with maximal deficiency sum// J. Anal. 27, 797-811 (2019).

29. A. Rathod. Characteristic function and deficiency of algebroid functions on annuli// Ufa Math. J. 11:1, 121-132 (2019).

30. A. Rathod. Value distribution of a algebroid function and its linear combination of derivatives on annuli // Electr. J. Math. Anal. Appl. 8:1, 129-142 (2020).

31. A. A. Kondratyuk, I. Laine. Meromorphic functions in multiply connected domains// in "Fourier Series Methods in Complex Analysis", Report Series. 10, Depart. Math. Univ. Joensuu, 1-111 (2006).

Ashok Rathod,

B.L.D.E.Association's

S.B. Arts and K.C.P. Science College,

Department of Mathematics,

SMT. Bangaramma Sajjan Campus,

Solapur Road, Vijayapura-586103,

Karnataka, India.

E-mail: ashokmrmaths@gmail.com 